identified as possible candidates for vaccination. Wasley et al. carried out a longitudinal analysis of reported incidences of hepatitis $A$ in the US since 1990. Incidence rates of hepatitis $A$ in 2003 for the country overall and in the 17 states where routine vaccination was recommended were compared with the equivalent rates during the prevaccination baseline period (1987-1997) that was used to establish the routine vaccination recommendations. Incidence rates in the 17 vaccinating states were also compared with the rates of all other states. The study showed that a dramatic decrease in the incidence of hepatitis $A$ of $76 \%$ had occurred since vaccination programs began (to 2.6 cases per 100,000). States that were selected for routine vaccination showed an $88 \%$ decrease in the incidence of hepatitis A (to 2.5 per 100,000), compared with $53 \%$ in nonvaccinating states (2.7 cases per 100,000). In 2003, states where routine vaccination was recommended accounted for $33 \%$ of hepatitis A cases compared with $65 \%$ before vaccination was introduced. An $87 \%$ decline in incidence was observed in children (2-18 years old); this was much greater than the $69 \%$ decline observed in persons above 18 years old. The proportion of all cases of hepatitis A occurring among children fell from $35 \%$ to $19 \%$. The highest rates of hepatitis $\mathrm{A}$ are now among young men (25-39 years old), and not children. In addition, the epidemiologic profile of patients with hepatitis A has changed substantially in the US following vaccinationthe large community-wide outbreaks that previously accounted for the majority of cases and which were driven primarily by infections among children have virtually disappeared, and there has been a shift in the relative frequency of reported sources of infection.

Dagan et al. examined the impact of a toddler vaccination program for hepatitis $A$ in Israel, which was implemented in 1999 for all children aged 18-24 months. Following implementation of the program, a dramatic decrease in the incidence of hepatitis $A$ was observed (95\% decrease since the preimmunization era $[\mathrm{P}<0.001])$, so that current rates are 2.2-2.5 cases per 100,000. This dramatic decline was most marked in children aged $1-4$ years $(98.2 \%$ decrease), but an overall decline of $>95 \%$ was shown for all age groups $(84.3 \%$ [ $>1$ year old], 96.5\% [5-9 years], 95.2\% [10-14 years], 91.3\% [15-44 years], 90.6\% [45-64 years] and $77.3 \%[\geq 65$ years $]$ ).
Further studies are required to confirm the efficacy of these vaccination strategies and to investigate the suggested herd immunity effect.

Rachel Jones

Original articles Wasley A et al. (2005) Incidence of hepatitis A in the United States in the era of vaccination. JAMA 294: 194-201

Dagan R et al. (2005) Incidence of hepatitis A in Israel following universal immunization of toddlers. JAMA 294: 202-210

\section{Overlap of chronic pelvic pain disorders and neural cross-talk}

Chronic pelvic pain disorders such as irritable bowel syndrome and interstitial cystitis often overlap in terms of symptoms and prevalence, and frequently occur concurrently. Pezzone and colleagues investigated the involvement of neural cross-talk in this overlap in a newly developed rodent model, and hypothesized that acute afferent irritation of one pelvic organ could sensitize another by means of shared or convergent afferent pathways.

The effects of urinary bladder irritation (performed by means of intravesical infusions) on sensory thresholds to colorectal distension, and of acute colonic irritation (with trinitrobenzene sulfonic acid) on bladder contraction frequency, were tested in urethrane-anesthetized female rats.

Electromyography measurements were taken. These demonstrated that irritation of the bladder triggered afferent colonic sensitization by means of an afferent reflex, as measured by sensitivity thresholds to graded colorectal distension. Following colonic irritation, the frequency of bladder contractions increased, suggesting that this organ had become irritated or sensitized.

The authors conclude that this is a viable model for use in further studies of pelvic inflammatory disorders and that their results provide strong evidence for bidirectional cross-sensitization of the colon and lower urinary tract. They suggest that the overlap observed between different chronic pelvic pain disorders might be a result of this cross-sensitization, and highlight the need for further research into these pathways.

Katy Cherry

Original article Pezzone MA et al. (2005) A model of neural cross-talk and irritation in the pelvis: implications for the overlap of chronic pelvic pain disorders. Gastroenterology 128: 1953-1964 